\title{
Notch Filter in 70 GHz Range for Microwave Plasma Diagnostics*)
}

\author{
Masaki NISHIURA, Shin KUBO, Kenji TANAKA, Sakuji KOBAYASHI, Kohta OKADA, \\ Teruki NISHIMURA, Koji OKADA, Hiroshi KASAHARA, Shinya OGASAWARA ${ }^{1)}$, \\ Takashi SHIMOZUMA, Takashi MUTOH, Kazuo KAWAHATA, Teruo SAITO2), \\ Yoshinori TATEMATSU ${ }^{2)}$ and LHD experiment group \\ National Institute for Fusion Science, 322-6 Oroshi-cho, Toki 509-5292, Japan \\ 1) Nagoya University, Furo-cho, Chikusa-ku, Nagoya 464-8601, Japan \\ 2) FIR FU, University of Fukui, 3-9-1 Bunkyo, Fukui 910-8507, Japan
}

(Received 7 December 2012 / Accepted 1 March 2013)

\begin{abstract}
A notch filter for the rejection of stray light from gigahertz range heating sources was developed to protect a vulnerable microwave plasma diagnostic system. As one of the applications, we consider the installation of the notch filter into the receiver of a collective Thomson scattering diagnostic in the Large Helical Device. Experimental observations indicate that two types of notch filters are required for main and spurious mode rejection; they have very narrow, steep shapes to avoid disturbing the diagnostic signal. On the basis of numerically simulated results, notch filters were fabricated, and their performance was evaluated. An attenuation level of $35 \mathrm{~dB}$ at $74.746 \mathrm{GHz}$ with a $3 \mathrm{~dB}$ bandwidth of $0.49 \mathrm{GHz}$ is achieved by two pairs of resonator cavities. This attenuation is acceptable in our study.
\end{abstract}

(c) 2013 The Japan Society of Plasma Science and Nuclear Fusion Research

Keywords: millimeter wave filters, electron cyclotron emission, reflectometer, collective Thomson scattering, millimeter plasma diagnostics

DOI: $10.1585 /$ pfr.8.2402027

\section{Introduction}

Fusion plasma diagnostics are essential for understanding plasma physics, controlling burning plasmas, and protecting vacuum vessels from disruptions or fast ion losses in fusion devices. One possible method of plasma heating and controlling is to use millimeter waves from megawatt power gyrotrons in electron cyclotron (EC) heating and current drive. However, microwave diagnostic devices, in particular those using electron cyclotron emission (ECE), microwave reflectometry, and collective Thomson scattering (CTS), suffer from stray light originating in the main mode radiation of a gyrotron inside the vacuum vessel, even if it becomes a small fraction. To protect microwave components from the stray light, a notch filter with a sharp, deep stop bandwidth can usually be applied.

These millimeter-wave diagnostics are typically used during EC heating to understand the underlying plasma transport. The noise radiation in the gyrotron radiation is mixed into the main mode radiation of the gyrotron. This spurious mode radiation from the gyrotron lies outside the frequency of a notch filter for the main mode radiation. For the CTS diagnostic, for example, the mixed spurious mode radiation in a probing beam should be excluded to protect the microwave components and reduce the interference signals. Some approaches have been considered to fix these problems. One approach is to optimize the gyrotron

author'se-mail: nishiura@nifs.ac.jp

*) This article is based on the presentation at the 22nd International Toki Conference (ITC22). operation to suppress the spurious mode output [1]. This decreases the gyrotron power; however, the power degradation is not suitable for plasma heating and the probing beam used in CTS diagnostics. Therefore, as another approach, we consider using a notch filter to protect a receiver from spurious mode radiation and reduce the superimposed noise.

In addition to these reasons, we have a possibility to develop a CTS diagnostic system with two sightlines. Thus, we need a notch filter for a new CTS receiver. The pass bandwidth of the present notch filter is limited to $+3 \mathrm{GHz}$ ( $80 \mathrm{GHz}$ in RF signal) from the nominal probing beam frequency of $76.95 \mathrm{GHz}$. It is desirable to extend this to $+5 \mathrm{GHz}(82 \mathrm{GHz})$ for a fast ion diagnostic.

A new gyrotron for plasma heating is recently operated in the Large Helical Device (LHD) experiment. It has a frequency of $154 \mathrm{GHz}$. We would like to use a notch filter for CTS or to protect the millimeter-wave receivers from the stray radiation. Our final goal is to fabricate a notch filter with a steep and very narrow rejection area, which is less than $200 \mathrm{MHz}$ around $77 \mathrm{GHz}$ or in the $154 \mathrm{GHz}$ range for main mode rejection with a total attenuation level of $120 \mathrm{~dB}$. We also need a notch filter for spurious mode rejection with an attenuation level of $30 \mathrm{~dB}$. In both cases, high manufacturing precision is required for these notch filters. We started by developing notch filters for spurious rejection to solve the present problems and to achieve relatively easy development because of the attenuation level. It 
is also advantageous because the area of the CTS spectrum removed by the notch filter is very narrow.

In this paper, we describe a design study using computer simulation software and the fabrication and characterization of notch filters for the reduction of spurious mode radiation with a narrow bandwidth from 74 to $80 \mathrm{GHz}$.

\section{Design Model for a Notch Filter}

Former studies showed that the spurious mode frequency between 74 and $80 \mathrm{GHz}$ is about $74.7 \mathrm{GHz}$ at the on and off timings of a $77 \mathrm{GHz}$ megawatt gyrotron during normal operation. As one example, the spectrogram measured by the CTS receiver for shot \#113377 is shown in Fig. 1. The spectrogram in three frequency regions is shown, and the raw signal before signal processing is plotted at the bottom. The probing beam power of the $77 \mathrm{GHz}$ gyrotron (L\#7) used for the CTS diagnostic is modulated at a frequency of $50 \mathrm{~Hz}$. The PIN switch, which can pass or intercept a signal within a few tens of nanoseconds, is driven at the rising and trailing edges to mask the strong spurious mode radiation. The spurious $\mathrm{TE}_{17,6}$ mode radiation can be masked at the rising edge but cannot be perfectly masked at the trailing edge. It is necessary to adjust the PIN switch timing and duration of a few milliseconds. The frequency of the $\mathrm{TE}_{17,6}$ mode radiation chirps down at the trailing edges. The chirping frequency should be covered by the notch filter's bandwidth. In addition, a spurious $\mathrm{TE}_{\mathrm{m}, \mathrm{n}}$ mode, whose mode number has not been identified, appears at a frequency region of $79.33-79.34 \mathrm{GHz}$. Although it does not significantly affect the raw signal of the

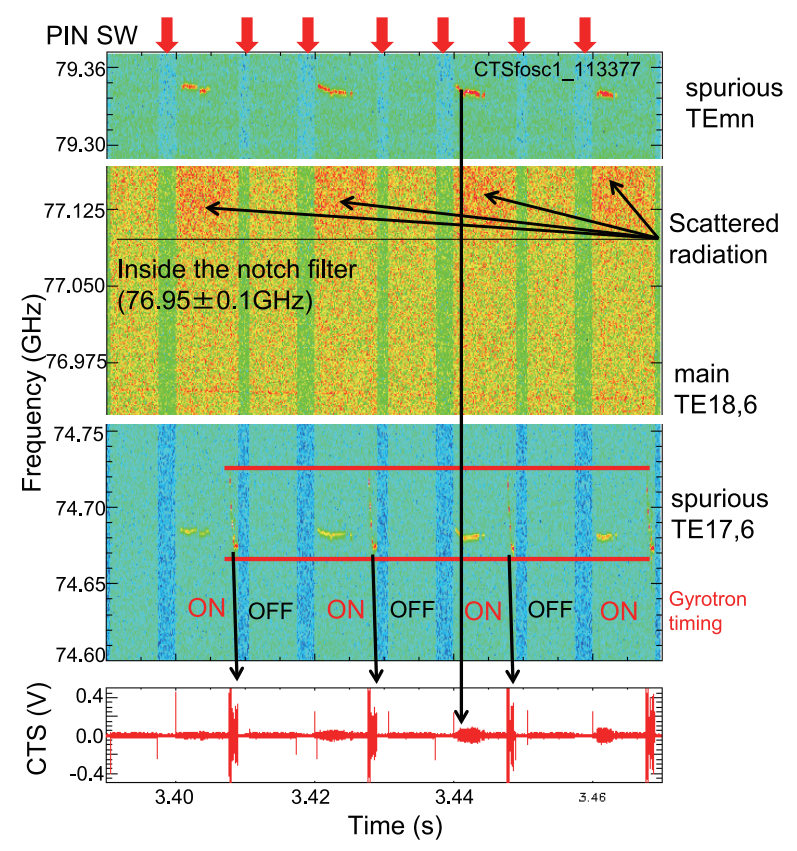

Fig. 1 Spectrogram of the gyrotron (L\#7) frequency measured by a high resolution CTS receiver during CTS experiments. fast digitizer, a notch filter must be used for a certain channel in the filter bank receiver.

A notch filter with a central frequency of $74.7 \mathrm{GHz}$ has been designed using the commercial software ANSYS HFSS. We simulated a waveguide-type notch filter that satisfied our specifications. For the narrow $3 \mathrm{~dB}$ bandwidth, mode control in resonator cavities is effective [2-5]. The main design parameters are listed in Table 1 . The $\mathrm{TE}_{11}$ mode is excited in the resonator cavities. The parameters determined here are noted. The distance between cavities $\Delta l$ is given by

$$
\Delta l=\frac{l_{\mathrm{g}}}{4}(1+2 N) .
$$

Here $N$ is an integer, and $\lambda_{\mathrm{g}}$ is the guide wavelength, which is introduced from the dispersion relation with the RF wavelength $\lambda_{0}$ as

$$
\lambda_{\mathrm{g}}=\frac{\lambda_{0}}{\sqrt{1-\left(\lambda_{0} / \lambda_{\mathrm{c}}\right)^{2}}} .
$$

The condition $\lambda_{\mathrm{c}}>\lambda_{0}$ is enforced. The cavity length $l$ is determined by

$$
l=\xi \frac{\lambda_{\mathrm{g}}}{2}
$$

where $\xi$ is an integer. The cut-off wavelength for the $\mathrm{TE}_{\mathrm{m}, \mathrm{n}}$ mode becomes

$$
\lambda_{\mathrm{c}, \mathrm{mn}}=\frac{\pi D}{v_{\mathrm{mn}}^{\prime}},
$$

where $v_{\mathrm{mn}}^{\prime}$ is the $n$th root of the derivative of the Bessel function $J_{\mathrm{m}}^{\prime}(x)=0$. The notch filter modeled here according to the determined parameters is shown in Fig. 2. Only two coupled cavities are shown in the model for simplicity. The design parameters, which mainly consist of the slit shape and cavity length, are adjusted to obtain the desired characteristics. The final design parameters are listed in Table 2. A bandwidth of $200 \mathrm{MHz}$ was ultimately achieved at the $3 \mathrm{~dB}$ rejection bandwidth by the simulation. The

Table 1 Design parameters of a notch filter for spurious rejection in $[\mathrm{mm}]$.

\begin{tabular}{|l|l|l|}
\hline broad side of waveguide & $\mathrm{a}$ & 3.099 \\
\hline RF wavelength & $\lambda_{0}$ & 4.013 \\
\hline cavity diameter & $\mathrm{D}$ & 3.000 \\
\hline cut-off wavelength & $\lambda_{\chi}$ & 6.198 \\
\hline cut-off wavelength for $\mathrm{TE}_{\mathrm{mn}}$ & $\lambda_{\mathrm{c}, 11}$ & 5.120 \\
\hline guide wavelength & $\lambda_{\mathrm{g}, 11}$ & 6.465 \\
\hline cavity length & $l$ & 3.232 \\
\hline & $2 l$ & 6.465 \\
\hline & $3 l$ & 9.697 \\
\hline $\begin{array}{l}\text { guide wavelength for main } \\
\text { waveguide }\end{array}$ & $\lambda_{\mathrm{g}}$ & 5.267 \\
\hline $\begin{array}{l}\text { Length between subsequent } \\
\text { cavities }\end{array}$ & $\Delta l$ & 3.950 \\
\hline
\end{tabular}




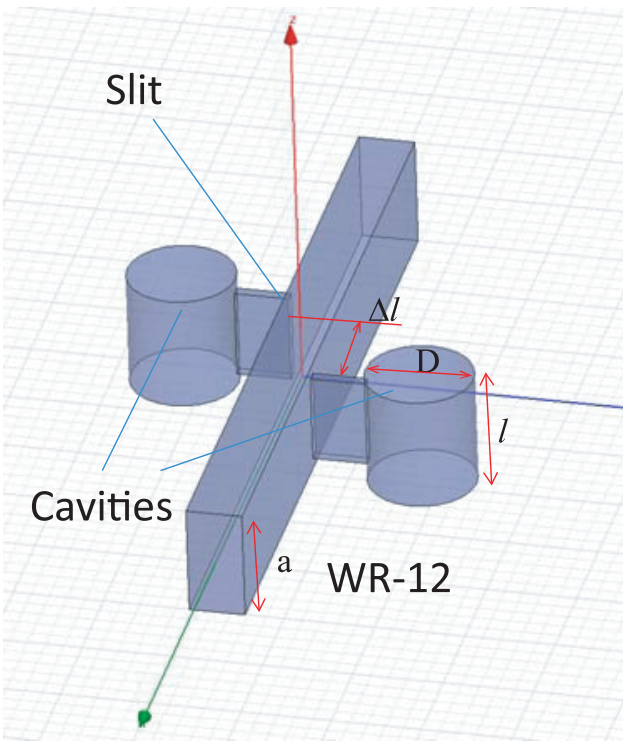

Fig. 2 3D model of a notch filter used in the study.

Table 2 Adjusted parameters and filter specifications obtained by simulation.

\begin{tabular}{|l|c|}
\hline & {$[\mathrm{mm}]$} \\
\hline Cavity length & 3.33 \\
\hline Slit width & 0.1 \\
\hline Slit length & 2.5 \\
\hline Slit depth & 0.6 \\
\hline$f$ & $74.75 \mathrm{GHz}$ \\
\hline$\delta f_{3 \mathrm{~dB}}$ & $0.2 \mathrm{GHz}$ \\
\hline Attenuation & $25 \mathrm{~dB}$ \\
\hline
\end{tabular}

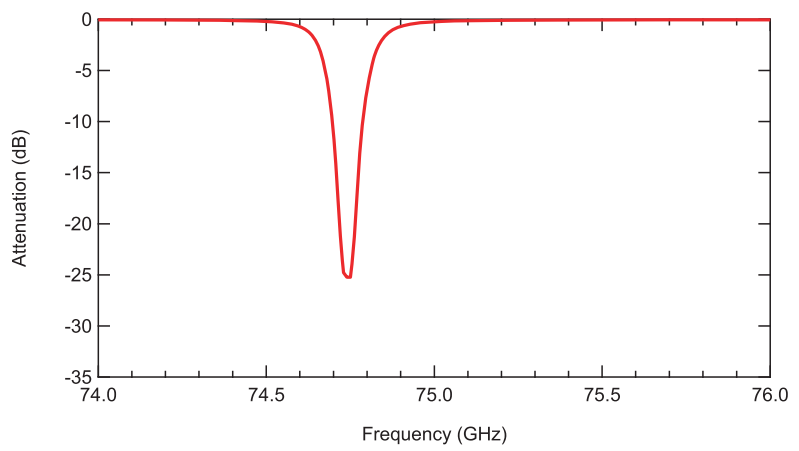

Fig. 3 Simulated characteristics of a notch filter for spurious rejection.

attenuation in the notch region was $25 \mathrm{~dB}$ with two resonant cavities, as shown in Fig. 3. We found that the cavity length changes the central frequency of the notch filter by around $0.01 \mathrm{~mm} / 150 \mathrm{MHz}$. To adjust the cavity length using screws, a smaller pitch is easier. A pitch of $0.34 \mathrm{~mm}$ is adopted.

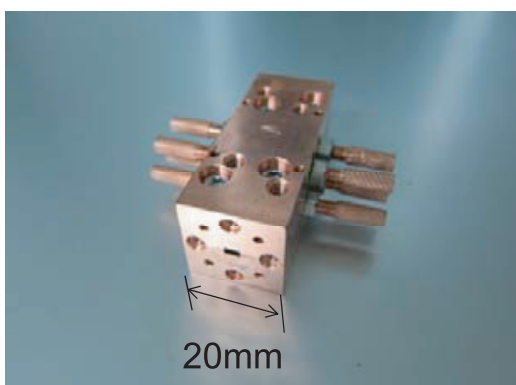

Fig. 4 Fabricated notch filter for spurious rejection. The cylindrical cavity lengths are adjusted from both ends by the screws visible in the photograph.
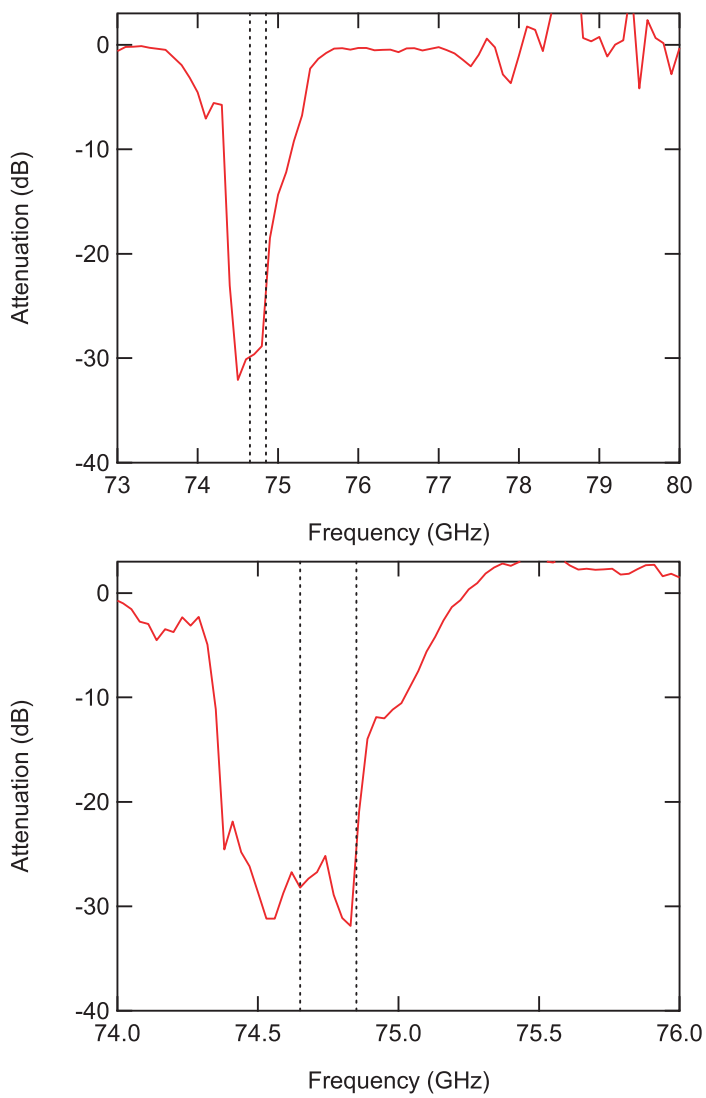

Fig. 5 Measured characteristics of the first trial notch filter. (a) Wide range and (b) near notch region.

\section{Notch Filter Fabrication}

After the design parameters were determined, the first trial piece was fabricated. The main body is a cuboid $(20 \times$ $20 \times 40 \mathrm{~mm}^{3}$ ), as shown in Fig. 4. A total of four cavities are arranged along the main waveguide, WR-12. Twice as many cavities as in the design model were adopted to obtain an attenuation of more than $40 \mathrm{~dB}$. The main material is oxide-free copper. Figure 5 shows the measured characteristics. The bandwidth of $200 \mathrm{MHz}$ is shown as broken lines in the graph. The measured $3 \mathrm{~dB}$ bandwidth is around $500 \mathrm{MHz}$, which is wider than the designed one. The difference was attributed to fabrication errors in the slit width 


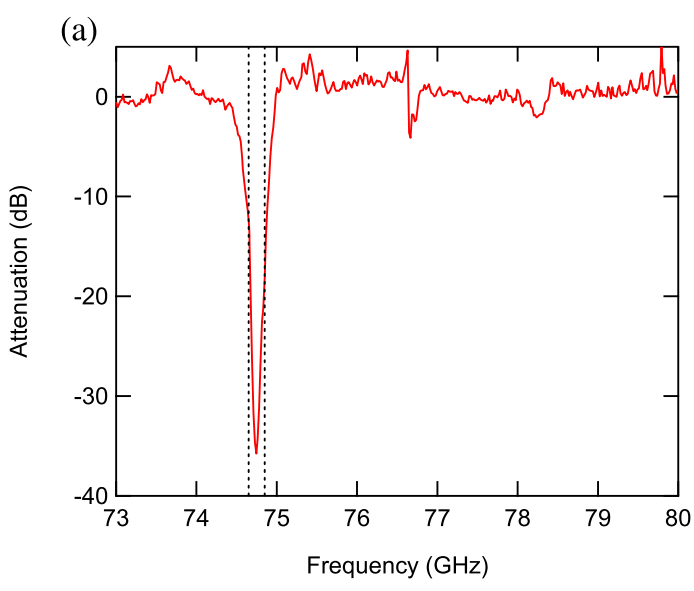

(b)

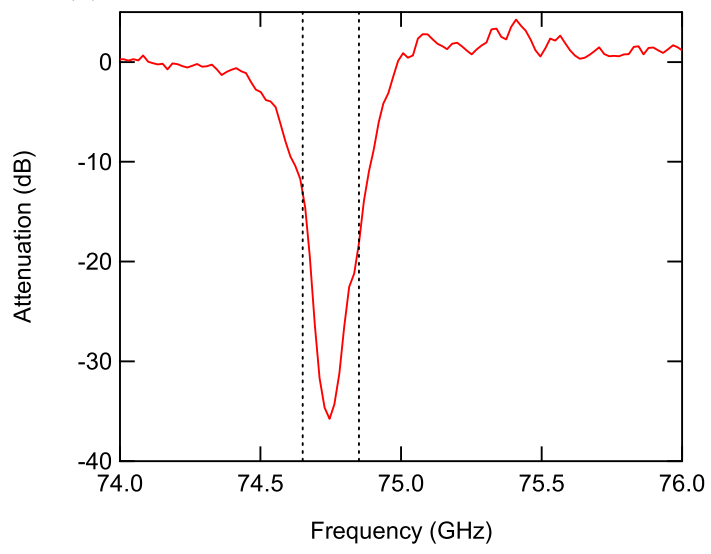

Fig. 6 Measured characteristics of the second trial notch filter. (a) Wide range and (b) near notch region.

and shape.

The second trial piece was carefully fabricated to realize a structure as close to the simulated one as possible. The measured filter characteristics are shown in Fig. 6. A $3 \mathrm{~dB}$ bandwidth of $0.49 \mathrm{GHz}$ and an attenuation of $35 \mathrm{~dB}$ were obtained at a central frequency of $74.746 \mathrm{GHz}$. The $3 \mathrm{~dB}$ bandwidth of the second trial piece is still wider than the design value. To examine this, the fabricated slit width and shape were measured by a digital microscope. The slit width was found to be $0.12 \mathrm{~mm}$, but the design value was $0.10 \mathrm{~mm}$. We simulated it again including a practical fabrication error of $\sim 0.02 \mathrm{~mm}$. The result shows an increase in the $3 \mathrm{~dB}$ bandwidth from 0.2 to $0.4 \mathrm{GHz}$. The measured bandwidth of $0.49 \mathrm{GHz}$ is still wider than this simulated value of $0.4 \mathrm{GHz}$.

Unexpected notches related to spurious modes were observed at 76.65 and $78.22 \mathrm{GHz}$. We believe that other $\mathrm{TE}_{\mathrm{m}, \mathrm{n}}$ modes are excited in the gap between the tuning plungers and the cylindrical cavity walls. As recommended in ref. [2], the insertion of attenuation rings in the cavities to absorb the spurious waves would be a good solution for the next trial.

\section{Summary}

We designed, fabricated, and characterized a 70-GHzrange notch filter for microwave plasma diagnostics. An attenuation level of $35 \mathrm{~dB}$ at $74.746 \mathrm{GHz}$ is achieved by two pairs of resonator cavities. This attenuation is acceptable in our study. The $3 \mathrm{~dB}$ bandwidth is $0.49 \mathrm{GHz}$, which is twice the design value. This is attributed to an increase in the slit width of $\sim 0.02 \mathrm{~mm}$ compared to the design value in fabrication. The unexpected spurious notches are expected to be reduced by improving the plunger structure, producing a flat passband and low insertion loss.

[1] S. Ogasawara, S. Kubo, M. Nishiura, Y. Tatematsu, T. Saito, K. Tanaka, T. Shimozuma, Y. Yoshimura, H. Igami, H. Takahashi, S. Ito, Y. Takita, S. Kobayashi, Y. Mizuno, K. Okada, R. Minami, T. Kariya and T. Imai, Rev. Sci. Instrum. 83, 10D731 (2012).

[2] T. Geist and M. Bergbauer, Int. J. Infrared Millim. Waves 15, 2043 (1994).

[3] V. Furtula, P.K. Michelsen, F. Leipold, M. Salewski, S.B. Korsholm, F. Meo, D. Moseev, S.K. Nielsen, M. Stejner and T. Johansen, Fusion Sci. Technol. 59, 670 (2011).

[4] V. Furtula, P.K. Michelsen, F. Leipold, M. Salewski, S.B. Korsholm, F. Meo, S.K. Nielsen, M. Stejner, D. Moseev and T. Johansen, Rev. Sci. Instrum. 81, 10D913 (2010).

[5] M. Iwase, K. Ohkubo, S. Kubo and H. Idei, J. Plasma Fusion Res. 72, 270 (1996). 